\title{
Identification and quantification of triacylglycerols containing n-3 long-chain polyunsaturated fatty acids in bovine milk
}

\author{
Zhiqian Liu, ${ }^{\star}$ Peter Moate, $\nmid$ Vilnis Ezerniks, ${ }^{*}$ Benjamin G. Cocks, ${ }^{*} \ddagger$ and Simone Rochfort ${ }^{\star} \ddagger^{1}$ \\ *Biosciences Research, Department of Economic Development, Jobs, Transport and Resources, AgriBio, 5 Ring Road, Bundoora, \\ Victoria 3083, Australia \\ †Farming Systems Research Division, Department of Economic Development, Jobs, Transport and Resources, Ellinbank Centre, \\ 1301 Hazeldean Rd, Ellinbank, Victoria 3821, Australia \\ $\ddagger$ School of Applied Systems Biology, La Trobe University, Bundoora, Victoria 3083, Australia
}

\section{ABSTRACT}

The n-3 long-chain polyunsaturated fatty acids (LCPUFA) are low-abundance components in milk fat, but have great potential in promoting human health. A comprehensive survey on triacylglycerol (TAG) molecular species in milk that contain at least one type of n-3 LCPUFA, namely eicosapentaenoic acid, docosahexaenoic acid, and docosapentaenoic acid, was conducted in this work using HPLC-linear trap quadrupole-Orbitrap and HPLC-triple quadrupole mass spectrometry techniques. A total of 51 TAG species that contain n-3 LC-PUFA have been identified in bovine milk and their structures assigned. The TAG species containing docosahexaenoic acid were found in much smaller number and at much lower abundance compared with the other 2 types of TAG. An HPLC-triple quadrupole mass spectrometrybased method was developed, which provides relative quantification of all these TAG species in a run of 36 min. Application of this method to the quantification of n-3 LC-PUFA-incorporated TAG in 32 individual animal milk samples allowed us to determine variation between animals, identify strong metabolic relationships between TAG species, and reveal negative effect of a grape marc supplement on the accumulation of eicosapentaenoic acid in milk.

Key words: milk, n-3 long-chain polyunsaturated fatty acids, liquid chromatography-mass spectrometry

\section{INTRODUCTION}

Although not all health benefit claims of n-3 FA have been substantiated, strong evidence exists that n-3 log-chain $(\geq \mathrm{C} 20)$ polyunsaturated fatty acids (n-3 LC-PUFA), especially eicosapentaenoic acid (EPA;

Received June 1, 2015.

Accepted August 9, 2015.

${ }^{1}$ Corresponding author: Simone.Rochfort@ecodev.vic.gov.au
C20:5n-3) and docosahexaenoic acid (DHA; C22:6n-3), have multiple beneficial functions for human health and development, such as prevention of cardiovascular disease, enhancing brain development in infants, and anti-inflammatory activity (Mori, 2006; Ruxton et al., 2007; Abeywardena and Patten, 2011). The n-3 LCPUFA are typically found in fish oils, which may not meet the rising demand for nutraceutical n-3 LC-PUFA products, thus an additional source of n-3 LC-PUFA is required (Nichols et al., 2010). Recently, algae have been cultivated to preferentially produce DHA and their oil has been extracted to make supplements (Sijtsma and de Swaaf, 2004). In addition, good progress has been made in metabolic engineering of n-3 LC-PUFA in oilseed crops by several groups in recently years (Wu et al., 2005; Cheng et al., 2010; Petrie et al., 2012). Breast milk is an important source of DHA and essential FA in early human life, but ruminant milk is known to be a poor source of n-3 LC-PUFA (Gastaldi et al., 2011). As milk is a staple drink and food ingredient, increasing its n-3 LC-PUFA content could increase the intake of such beneficial FA for a large number of people. Recently it has been shown that the concentrations of n-3 LC-PUFA in cow milk can be substantially increased by supplementing the cow diet with feed containing high quantities of n-3 FA (Moghadasian, 2008; Nelson and Martini, 2009; Moate et al., 2013). One study also found that the concentration of n-3 FA, including EPA and docosapentaenoic acid (DPA; C22:5n-3), was higher in organic milk as compared with conventional milk (Benbrook et al., 2013). A recent study into the heritability of lipid classes in milk (SFA, MUFA, and PUFA) suggests that potential to select for a healthy milk composition exists (Penasa et al., 2015). Underpinning any genetic or management strategy to change the composition of milk lipids is the ability to accurately measure the lipid species.

The global FA composition of any food sample can be readily measured by transesterification and GC analysis (Slover and Lanza, 1979). Consequently, numerous 
data can be found regarding the FA profile of bovine milk fat (Moate et al., 2007, 2014; Mansson, 2008; Frelich et al., 2009; Gastaldi et al., 2011); the major FA species are consistent, which include C4, C6, C8, C10, C12, C14, C14:1, C16, C16:1, C18, C18:1, C18:2, and C18:3. However, the concentration of n-3 LC-PUFA was not given in most reports, presumably due to the low abundance of these FA, which were below the limit of quantification in those studies.

Currently, information on the molecular species and the relative abundance of triacylglycerol (TAG) that contain n-3 LC-PUFA in milk fat is also scarce, although detailed profiling of the relatively abundant TAG species has been reported extensively (Gresti et al., 1993; Mottram and Evershed, 2001; Månsson, 2008; Gastaldi et al., 2011; Haddad et al., 2011; Zhou et al., 2014a). To investigate the accumulation pattern of n-3 LC-PUFA in milk fat in relation to various genetic and environmental factors, a systematic characterization of the TAG species that contain n-3 LC-PUFA as well as a robust method for their quantification is required.

Grape marc (skins, seeds, and stems that remain after grapes are pressed for juice-making), is currently a by-product used as a feed supplement by the dairy and beef industries. A recent study showed that grape marc could reduce methane emissions when fed to dairy cow, indicating that this by-product could play a role in methane abatement (Moate et al., 2014). The influence of grape marc diet on the concentration of major FA of milk fat has been investigated in the same study (Moate et al., 2014). However, whether lowabundance n-3 LC-PUFA is affected by grape marc diet remained unknown. The objectives of our study were (1) comprehensive identification of molecular species of TAG that contain EPA, DHA, or DPA; (2) developing a HPLC-triple quadrupole MS-based method for simultaneous quantification of the identified n-3 LCPUFA-incorporated TAG species; (3) investigating the variance in abundance of $n-3$ LC-PUFA-containing TAG species between cows; and (4) determining of the effect of grape marc diet on the accumulation of $n-3$ LC-PUFA in milk.

\section{MATERIALS AND METHODS}

\section{Cows, Diets, and Milk Samples}

Thirty-two lactating, multiparous Holstein-Friesian cows $(500 \pm 51.9 \mathrm{~kg}$ of BW, $22.1 \pm 5.1 \mathrm{DIM}, 3.4 \pm$ $0.50 \mathrm{yr}$ of age; average $\pm \mathrm{SD}$ ) were assigned to 3 groups balanced for BW, DIM, and age. Each group was then randomly allocated to 1 of 3 dietary treatments: (1) a control diet $(\mathbf{C O N})$ in which cows were individually offered $5.0 \mathrm{~kg}$ of DM of cracked corn, $0.2 \mathrm{~kg}$ of DM of minerals, and $15.0 \mathrm{~kg}$ of DM of freshly cut pasture (predominantly ryegrass); (2) white-grape marc diet (WGM) in which cows were individually offered 5.0 $\mathrm{kg}$ of DM of cracked corn, $0.2 \mathrm{~kg}$ of DM of minerals, $5.0 \mathrm{~kg}$ of DM of white-grape marc, and $10.0 \mathrm{~kg}$ of DM of freshly cut pasture; and (3) a red-grape marc diet (RGM) in which cows were individually offered 5.0 $\mathrm{kg}$ of DM of cracked corn, $0.2 \mathrm{~kg}$ of DM of minerals, $5.0 \mathrm{~kg}$ of DM of red-grape marc, and $10.0 \mathrm{~kg}$ of DM of freshly cut pasture. The CON treatment had 12 cows assigned to it, whereas the WGM and RGM treatments each had 10 cows. These diets were fed to the cows for $28 \mathrm{~d}$ and samples of milk were collected on the last day of the experiment and kept at $-80^{\circ} \mathrm{C}$ before analysis.

\section{Chemicals}

The n-3 LC-PUFA methyl ester standards, TAG triC22:1 (used as internal standard; IS), and ammonium formate ( $\geq 99 \%$ purity, used as a mobile phase additive) were purchased from Sigma Aldrich (St. Louis, MO). Solvents used for lipid extraction and mobile phase preparation were of chromatographic grade and were from Merck (methanol; Merck, Kenilworth, NJ) and Sigma Aldrich (chloroform, isopropanol, and acetonitrile containing $0.1 \%$ formic acid). Sulfuric acid used in lipid transesterification was of analytical grade (Ajax Finechem, Seven Hills, Australia).

\section{Method for Lipid Extraction from Milk}

Milk lipid was extracted as described in our previous report (Liu et al., 2015). Briefly, $0.2 \mathrm{~mL}$ of full-cream milk, $50 \mu \mathrm{L}$ of IS (TAG tri-C22:1, $0.1 \mathrm{mg} / \mathrm{mL}$ in chloroform and methanol at a 2:1 ratio), and $0.8 \mathrm{~mL}$ of MilliQ water (Merck Millipore, Billerica, MA) was added; the diluted milk was mixed with $4 \mathrm{~mL}$ of chloroform and methanol $(2: 1, \mathrm{vol} / \mathrm{vol})$ and shaken in a vortex mixer thoroughly for $10 \mathrm{~min}$ at room temperature. The mixture was then centrifuged for 10 min at 1,900 $\times g$ at room temperature to facilitate phase separation. After transferring the organic phase to a new tube, the aqueous phase was extracted again with $2 \mathrm{~mL}$ of chloroform and methanol $(2: 1, \mathrm{vol} / \mathrm{vol})$. The combined organic phase (ca. $4.5 \mathrm{~mL}$ ), which contained the lipid fraction, was dried under a stream of nitrogen and reconstituted at a $3: 1$ ratio in isopropanol and chloroform $(2: 1, \mathrm{vol} /$ vol) before analysis by HPLC-MS.

\section{Method for Identification and Quantification of n-3 LC-PUFA-Containing TAG}

Two types of HPLC-MS configurations were employed in our study: HPLC-linear trap quadrupole 
(LTQ)-Orbitrap MS and HPLC-triple quadrupole MS. The same HPLC conditions were used in both cases. Chromatographic separation of TAG was achieved using a Synergi Polar-RP column $(250 \times 4.6 \mathrm{~mm}, 4 \mu \mathrm{m}$, Phenomenex, Torrance, CA) on an Agilent 1290 Infinity HPLC system (Agilent Technologies, Santa Clara, $\mathrm{CA}$ ), including degasser, binary pump, temperaturecontrolled autosampler (maintained at $10^{\circ} \mathrm{C}$ ), and column compartment (maintained at $55^{\circ} \mathrm{C}$ ). The mobile phase was composed of $5 \mathrm{~m} M$ aqueous ammonium formate $(\mathrm{A})$ and acetonitrile $+0.1 \%$ formic acid and isopropanol $(1: 1, \mathrm{vol} / \mathrm{vol} ; \mathrm{B})$. The flow rate was 0.6 $\mathrm{mL} / \mathrm{min}$ with a gradient elution of 75 to $98 \%$ B over $25 \mathrm{~min}$. The injection volume was $5 \mu \mathrm{L}$ for all analyses.

For the identification of TAG species that contain at least 1 of the 3 n-3 LC-PUFA, a neutral loss (NL) scan (550-1,000 $\mathrm{m} / \mathrm{z}$ ) was performed separately for EPA $(\mathrm{NL}=319 \mathrm{amu})$, DHA $(\mathrm{NL}=345 \mathrm{amu})$, and DPA $(\mathrm{NL}=347 \mathrm{amu})$ residues on HPLC-Triple Quadrupole MS in positive ion mode. The MS settings included a fragmentor voltage of $150 \mathrm{~V}$, a collision energy of $25 \mathrm{~V}$, and a spray voltage of $4.2 \mathrm{KV}$. The putative n-3 LC-PUFA-containing TAG species identified by NL scan were further verified on the LTQ-Orbitrap MS operated with a full scan in Fourier transform (FT) positive ion mode at a resolution of 60,000 , followed by a data-dependent MS/MS scan in ion trap mode with a normalized collision energy of 25 arbitrary units. Only those precursor ions that matched the calculated accurate mass $( \pm 10 \mathrm{ppm})$ and expected MS/MS fragmentation pattern of TAG molecules were retained and their structure assigned. Subsequently, the confirmed n-3 LC-PUFA-containing TAG species were quantified on the triple quadrupole MS in multiple reaction monitoring (MRM) positive ion mode. The results are presented as relative abundance of individual TAG species across different milk samples (after normalization by IS). For both MS instruments, molecular ions of TAG were detected as ammonium adducts.

\section{Method for Quantification of n-3 LC-PUFA Methyl Esters in Milk by LC-MS}

Milk lipid was extracted by the method of Bligh and Dyer (1959). After removal of chloroform under a stream of nitrogen, the transesterification of the extracted lipids was carried out by adding acidic methanol $(6 \%$ $\mathrm{H}_{2} \mathrm{SO}_{4}$ ) and heating at $80^{\circ} \mathrm{C}$ for $3 \mathrm{~h}$ (Taylor and Savage, 2006). The released FAME were extracted by hexane and then reconstituted at a 3:1 ratio in isopropanol and chloroform (2:1, vol/vol) before analysis by HPLC-MS.

The separation of FAME was carried out with a Poroshell 120 EC-C18 column $(150 \times 4.6 \mathrm{~mm}, 2.7 \mu \mathrm{m}$, Agilent Technologies). The mobile phase was composed of $5 \mathrm{~m} M$ aqueous ammonium formate (A) and acetonitrile $+0.1 \%$ formic acid (B). The flow rate was 0.4 $\mathrm{mL} / \mathrm{min}$ with a gradient elution of 85 to $100 \%$ B over 15 min. Analyte detection was by LTQ-Orbitrap MS in FT positive ion mode (at a resolution of 60,000). Protonated ions of DHA, EPA, and DPA methyl esters were extracted from the full scan chromatograms and peak areas integrated using Xcalibur software (Thermo Fisher Scientific, Waltham, MA). The concentration of the 3 types of n-3 LC-PUFA methyl esters was determined using external standard calibration.

\section{Statistical Analysis of Results}

All TAG and n-3 LC-PUFA methyl ester quantification results obtained from the grape marc feeding experiment were subjected to ANOVA; where significant differences were found between diet treatments, a Student's $t$-test was conducted for pairwise comparison with Bonferroni correction applied. Both ANOVA and $t$-test were performed with Microsoft Excel 2010 (Microsoft Corp., Redmond, WA). Principal component analysis for unsupervised classification of samples was performed with MATLAB R2014a (MathWorks, Natick, MA) utilizing PLS Toolbox (Eigenvector Research, Manson, WA).

\section{RESULTS}

\section{Identification of n-3 LC-PUFA-Containing TAG Species in Milk}

An NL scan was conducted separately for the loss of EPA (C20:5), DHA (C22:6), and DPA (C22:5) FA moieties using the same fragmentor $(150 \mathrm{~V})$ and collision energy $(25 \mathrm{~V})$ settings. An example of a total ion chromatogram from one NL scan (for an NL of 319 amu, i.e., EPA) is shown in Figure 1.

A large number of peaks (precursor ions) were identified for each of the 3 types of NL. These precursors may be from different lipid classes that contain n-3 LC-PUFA (such as TAG, diacylglycerols, phospholipids, and so on) or from nonlipid compounds that can afford the same nominal NL. As the current study was aimed at the characterization of n-3 LC-PUFA-containing TAG species, non-TAG compounds were not considered.

High-resolution Orbitrap MS allowed us to determine the accurate mass of all candidate precursors identified by NL scan; only those with a mass accuracy difference of less than $10 \mathrm{ppm}$ (compared with the theoretical values of TAG) were retained. These putative n-3 LCPUFA-containing TAG species were further verified by examining their MS/MS profile for expected product ions (after an NL); the MS/MS fragmentation pattern 
was also used to assign the structure of the TAG species.

For example, a precursor ion with a nominal mass of 924.7 was identified by 2 different NL scans (C20:5 and C22:5) on triple quadrupole MS. This precursor ion was then subjected to verification by Orbitrap MS and was found to have an accurate mass of 924.8009 , which was $1.2 \mathrm{ppm}$ different from a TAG molecular feature (formula $=\mathrm{C}_{59} \mathrm{H}_{102} \mathrm{O}_{6}$; accurate mass $=924.802$ ). The MS/MS profile of this precursor is shown in Figure 2. The 2 diacylglycerol ions $(m / z: 577.5$ and 605.5$)$ and the $2 \mathrm{FA}$ acylium ions $(\mathrm{m} / \mathrm{z}: 313$ and 285$)$ confirmed the presence of C20:5 (EPA) and C22:6 (DPA) in this TAG molecule. The total carbon number and the total double bond number of the FA moieties (C56:6) suggests that this TAG molecule cannot contain both EPA and DPA, so the only interpretation for this MS/MS spectrum is that the molecule is present as 2 coeluting structural isomers with each containing a different n-3 LC-PUFA. Based on the whole diacylglycerol ion spectrum, the most likely structures for these 2 TAG isomeric species are $\mathrm{C} 16-\mathrm{C} 18: 1-\mathrm{C} 22: 5$ and $\mathrm{C} 18-\mathrm{C} 18: 1-$ C20:5, respectively. Using this approach, a total of 51 TAG species from 24 molecular features have been confirmed as containing 1 of the 3 n-3 LC-PUFA. The molecular features (accurate mass, total carbon number, and total double bond number of the FA moieties) and the structures of these TAG species are summarized in Table 1.

Among the 51 TAG species, only 10 contain DHA, whereas a larger number of species containing EPA or DPA (20 and 21, respectively) were identified in bovine milk. Close examination of Table 1 reveals that although the majority of the species are composed of

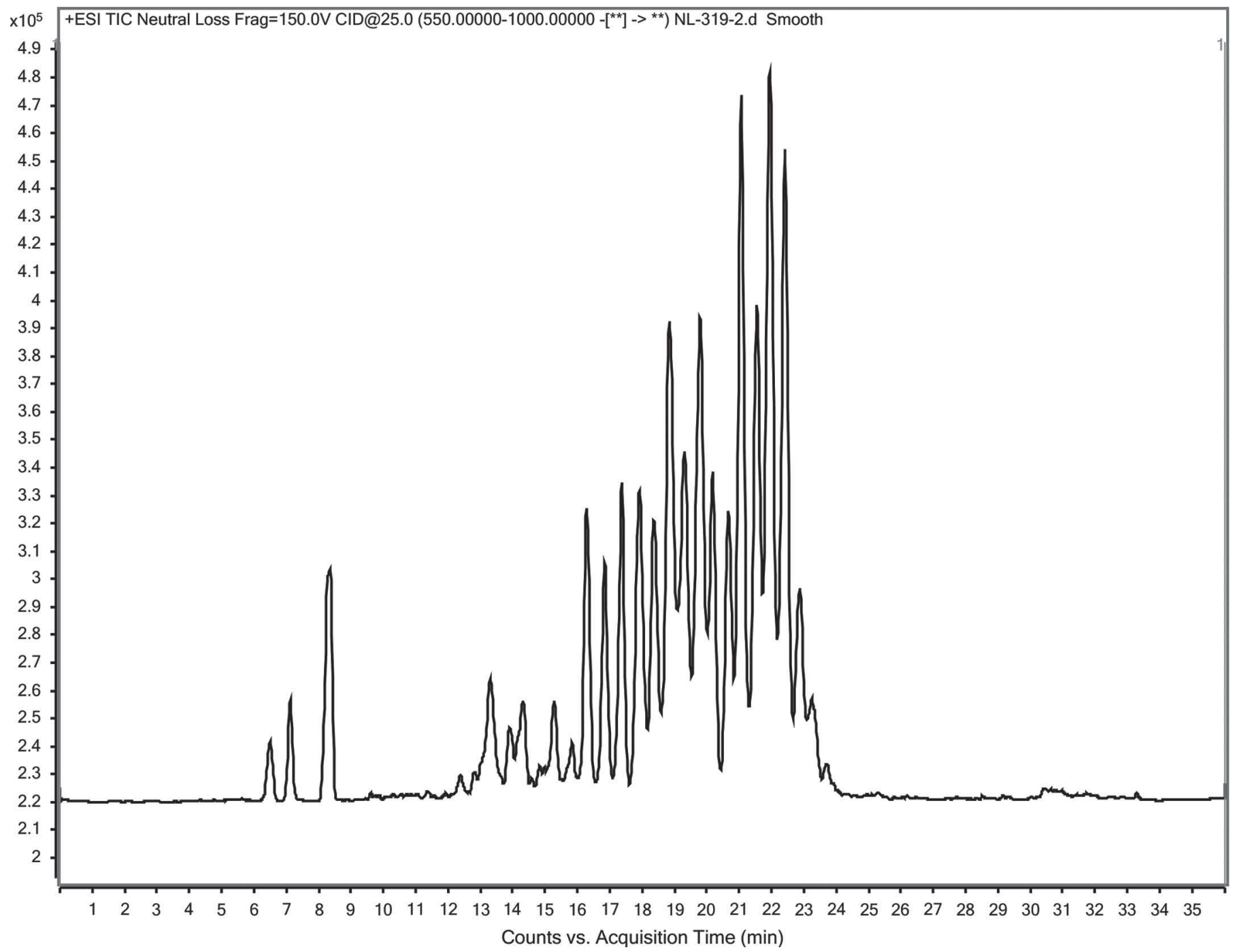

Figure 1. Total ion chromatogram of a neutral loss (NL) scan (NL: $319 \mathrm{amu}$ of eicosapentaenoic acid). 
even-numbered FA, 1 odd-numbered SFA (C15) was found in 3 species (EPA-13, EPA-16, and DPA-12). All n-3 LC-PUFA were esterified together with either 2 medium-chain FA (C10-C18), or 1 short-chain (C4$\mathrm{C} 8$ ) and 1 medium-chain FA. In addition, all the 51 species were found to have a molecular mass between 672 and 952; likewise, for any TAG molecular feature within this mass range that has a total double bond number $\geq 5$, at least 1 species containing 1 of the 3 n- 3 LC-PUFA can be found.

\section{Quantification of n-3 LC-PUFA-Containing TAG Species}

The 51 TAG species belonging to 3 n-3 LC-PUFA types were quantified using HPLC-triple quadrupole
MS in MRM mode (1 s/cycle). The specific product ion (i.e., the diacylglycerol ion after NL of the corresponding n-3 LC-PUFA) selected and the collision energy used for quantification of each species are summarized in Supplemental Table S1 (http://dx.doi.org/10.3168/ jds.2015-9889). A total ion chromatogram of all the transitions and an example of 6 selected MRM profiles of a milk sample are shown in Figure 3. Again for each of the 2 precursor ions $(m / z: 812.6$ and 840.7$), 3$ structural isomers each containing a different n-3 LC-PUFA were identified. These coeluting isomeric species can be reliably quantified using a specific product ion.

After verification of its measurement reproducibility (relative SD of peak area for 3 repeated analyses $<5 \%$ for all transitions from a random sample, results not shown), this method was applied to the quantification

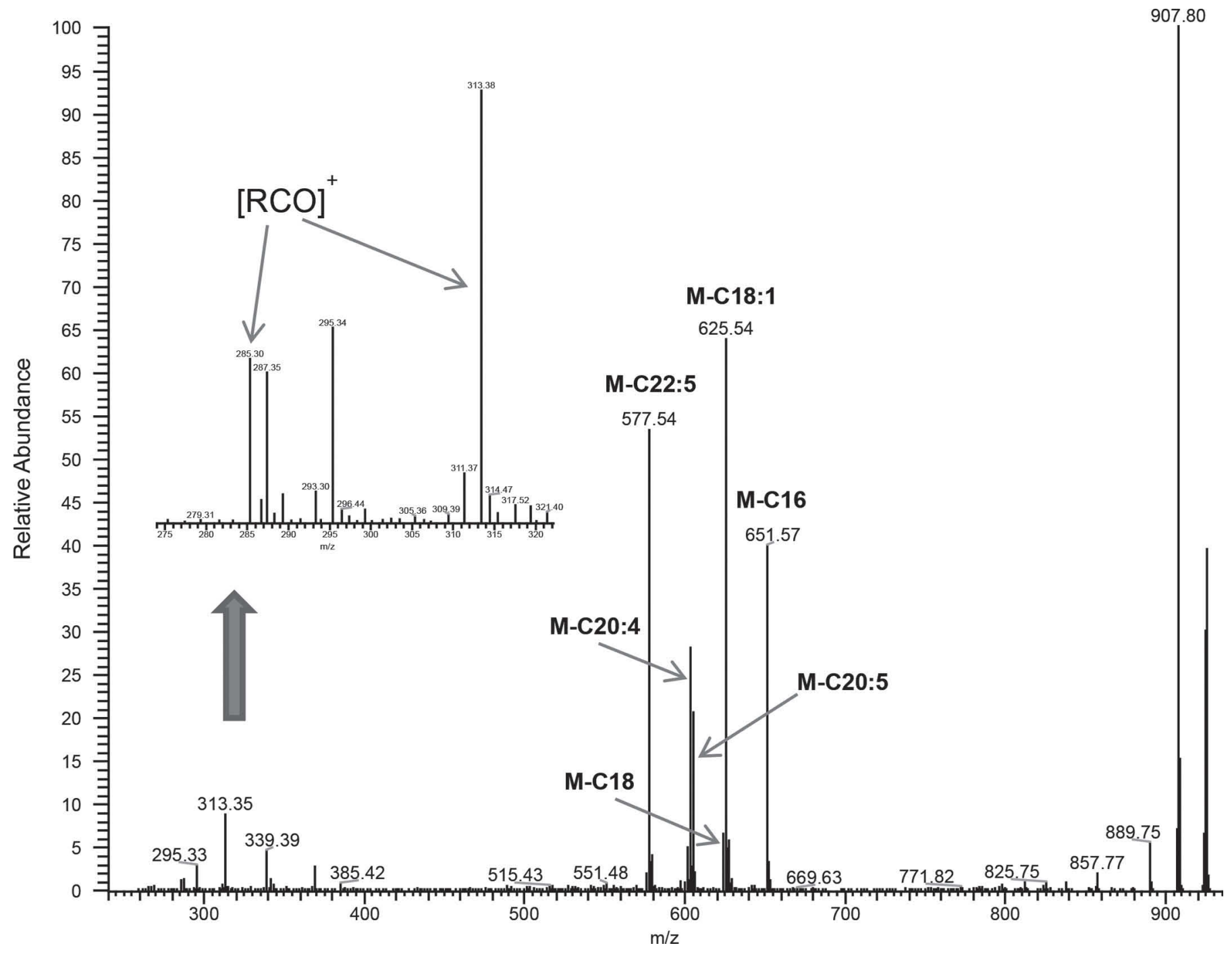

Figure 2. The MS/MS profile of 2 coeluted n-3 long-chain PUFA-containing triacylglyceride isomers. $[\mathrm{RCO}]^{+}=$fatty acid acylium ion; $\mathrm{M}$ $=$ molecular ion 
of the 51 TAG species in 32 milk samples collected from control and grape marc-fed cows. The results are summarized in Figure 4.

This comprehensive data set allows us to reveal the overall distribution pattern of the 3 types of n-3 LCPUFA in milk TAG: (1) the DHA-containing TAG species are not only found in smaller number, but also in lower abundance compared with the other 2 n-3 LCPUFA types; and (2) within each n-3 LC-PUFA type, large variation in abundance is observed across different species, suggesting a nonrandom incorporation of n-3 LC-PUFA into TAG molecules.

Table 1. List of triacylglyceride species containing n-3 long-chain PUFA identified

\begin{tabular}{|c|c|c|c|c|}
\hline Code $^{1}$ & $m / z{\text { (calculated })^{2}}^{2}$ & Formula & $\mathrm{CN}: \mathrm{DB}^{3}$ & Structure \\
\hline EPA-1 & 674.5362 & $\mathrm{C}_{41} \mathrm{H}_{68} \mathrm{O}_{6}$ & $38: 5$ & C4-C14-C20:5 \\
\hline EPA-2 & 702.5675 & $\mathrm{C}_{43} \mathrm{H}_{72} \mathrm{O}_{6}$ & $40: 5$ & C4-C16-C20:5 \\
\hline EPA-3 & 728.5831 & $\mathrm{C}_{45} \mathrm{H}_{74} \mathrm{O}_{6}$ & $42: 6$ & C4-C18:1-C20:5 \\
\hline EPA-4 & 730.5987 & $\mathrm{C}_{45} \mathrm{H}_{76} \mathrm{O}_{6}$ & $42: 5$ & C6-C16-C20:5 \\
\hline EPA-5 & 756.6145 & $\mathrm{C}_{47} \mathrm{H}_{78} \mathrm{O}_{6}$ & $44: 6$ & C6-C18:1-C20:5 \\
\hline EPA-6 & 758.6301 & $\mathrm{C}_{47} \mathrm{H}_{80} \mathrm{O}_{6}$ & $44: 5$ & C8-C16-C20:5 \\
\hline EPA-7 & 784.6457 & $\mathrm{C}_{49} \mathrm{H}_{82} \mathrm{O}_{6}$ & $46: 6$ & C10:1-C16-C20:5 \\
\hline EPA-8 & 786.6613 & $\mathrm{C}_{49} \mathrm{H}_{84} \mathrm{O}_{6}$ & $46: 5$ & C10-C16-C20:5 \\
\hline EPA-9 & 812.6770 & $\mathrm{C}_{51} \mathrm{H}_{86} \mathrm{O}_{6}$ & $48: 6$ & C10-C18:1-C20:5 \\
\hline EPA-10 & 814.6926 & $\mathrm{C}_{51} \mathrm{H}_{88} \mathrm{O}_{6}$ & $48: 5$ & C12-C16-C20:5 \\
\hline EPA-11 & 840.7081 & $\mathrm{C}_{53} \mathrm{H}_{90} \mathrm{O}_{6}$ & $50: 6$ & C12-C18:1-C20:5 \\
\hline EPA-12 & 842.7238 & $\mathrm{C}_{53} \mathrm{H}_{92} \mathrm{O}_{6}$ & $50: 5$ & C14-C16-C20:5 \\
\hline EPA-13 & 856.7395 & $\mathrm{C}_{54} \mathrm{H}_{94} \mathrm{O}_{6}$ & $51: 5$ & C15-C16-C20:5 \\
\hline EPA-14 & 868.7394 & $\mathrm{C}_{55} \mathrm{H}_{94} \mathrm{O}_{6}$ & $52: 6$ & C14-C18:1-C20:5 \\
\hline EPA-15 & 870.7551 & $\mathrm{C}_{55} \mathrm{H}_{96} \mathrm{O}_{6}$ & $52: 5$ & C16-C16-C20:5 \\
\hline EPA-16 & 882.7553 & $\mathrm{C}_{56} \mathrm{H}_{96} \mathrm{O}_{6}$ & $53: 6$ & C15-C18:1-C20:5 \\
\hline EPA-17 & 894.7551 & $\mathrm{C}_{57} \mathrm{H}_{96} \mathrm{O}_{6}$ & $54: 7$ & C16-C18:2-C20:5 \\
\hline EPA-18 & 896.7707 & $\mathrm{C}_{57} \mathrm{H}_{98} \mathrm{O}_{6}$ & $54: 6$ & C16-C18:1-C20:5 \\
\hline EPA-19 & 922.7864 & $\mathrm{C}_{59} \mathrm{H}_{100} \mathrm{O}_{6}$ & $56: 7$ & C18:1-C18:1-C20:5 \\
\hline EPA-20 & 924.8020 & $\mathrm{C}_{59} \mathrm{H}_{102} \mathrm{O}_{6}$ & $56: 6$ & C18-C18:1-C20:5 \\
\hline DPA-1 & 702.5675 & $\mathrm{C}_{43} \mathrm{H}_{72} \mathrm{O}_{6}$ & $40: 5$ & C4-C14-C22:5 \\
\hline DPA-2 & 728.5831 & $\mathrm{C}_{45} \mathrm{H}_{74} \mathrm{O}_{6}$ & $42: 6$ & C4-C16:1-C22:5 \\
\hline DPA-3 & 730.5987 & $\mathrm{C}_{45} \mathrm{H}_{76} \mathrm{O}_{6}$ & $42: 5$ & C4-C16-C22:5 \\
\hline DPA-4 & 756.6145 & $\mathrm{C}_{47} \mathrm{H}_{78} \mathrm{O}_{6}$ & $44: 6$ & C4-C18:1-C22:5 \\
\hline DPA-5 & 758.6301 & $\mathrm{C}_{47} \mathrm{H}_{80} \mathrm{O}_{6}$ & $44: 5$ & C6-C16-C22:5 \\
\hline DPA- 6 & 784.6457 & $\mathrm{C}_{49} \mathrm{H}_{82} \mathrm{O}_{6}$ & $46: 6$ & C6-C18:1-C22:5 \\
\hline DPA-7 & 786.6613 & $\mathrm{C}_{49} \mathrm{H}_{84} \mathrm{O}_{6}$ & $46: 5$ & $\mathrm{C} 8-\mathrm{C} 16-\mathrm{C} 22: 5$ \\
\hline DPA-8 & 812.6770 & $\mathrm{C}_{51} \mathrm{H}_{86} \mathrm{O}_{6}$ & $48: 6$ & C10:1-C16-C22:5 \\
\hline DPA-9 & 814.6926 & $\mathrm{C}_{51} \mathrm{H}_{88} \mathrm{O}_{6}$ & $48: 5$ & C10-C16-C22:5 \\
\hline DPA-10 & 840.7081 & $\mathrm{C}_{53} \mathrm{H}_{90} \mathrm{O}_{6}$ & $50: 6$ & C10-C18:1-C22:5 \\
\hline DPA-11 & 842.7238 & $\mathrm{C}_{53} \mathrm{H}_{92} \mathrm{O}_{6}$ & $50: 5$ & $\mathrm{C} 12-\mathrm{C} 16-\mathrm{C} 22: 5$ \\
\hline DPA-12 & 856.7395 & $\mathrm{C}_{54} \mathrm{H}_{94} \mathrm{O}_{6}$ & $51: 5$ & C14-C15-C22:5 \\
\hline DPA-13 & 868.7394 & $\mathrm{C}_{55} \mathrm{H}_{94} \mathrm{O}_{6}$ & $52: 6$ & C14:1-C16-C22:5 \\
\hline DPA-14 & 870.7551 & $\mathrm{C}_{55} \mathrm{H}_{96} \mathrm{O}_{6}$ & $52: 5$ & C14-C16-C22:5 \\
\hline DPA-15 & 896.7707 & $\mathrm{C}_{57} \mathrm{H}_{98} \mathrm{O}_{6}$ & $54: 6$ & C14-C18:1-C22:5 \\
\hline DPA-16 & 898.7864 & $\mathrm{C}_{57} \mathrm{H}_{100} \mathrm{O}_{6}$ & $54: 5$ & $\mathrm{C} 16-\mathrm{C} 16-\mathrm{C} 22: 5$ \\
\hline DPA-17 & 922.7864 & $\mathrm{C}_{59} \mathrm{H}_{100} \mathrm{O}_{6}$ & $56: 7$ & C16-C18:2-C22:5 \\
\hline DPA-18 & 924.8020 & $\mathrm{C}_{59} \mathrm{H}_{102} \mathrm{O}_{6}$ & $56: 6$ & C16-C18:1-C22:5 \\
\hline DPA-19 & 926.8177 & $\mathrm{C}_{59} \mathrm{H}_{104} \mathrm{O}_{6}$ & $56: 5$ & C16-C18-C22:5 \\
\hline DPA-20 & 950.8177 & $\mathrm{C}_{61} \mathrm{H}_{104} \mathrm{O}_{6}$ & $58: 7$ & C18:1-C18:1-C22:5 \\
\hline DPA-21 & 952.8333 & $\mathrm{C}_{61} \mathrm{H}_{106} \mathrm{O}_{6}$ & $58: 6$ & C18-C18:1-C22:5 \\
\hline DHA-1 & 728.5831 & $\mathrm{C}_{45} \mathrm{H}_{74} \mathrm{O}_{6}$ & $42: 6$ & C4-C16-C22:6 \\
\hline DHA-2 & 756.6145 & $\mathrm{C}_{47} \mathrm{H}_{78} \mathrm{O}_{6}$ & $44: 6$ & C6-C16-C22:6 \\
\hline DHA-3 & 784.6457 & $\mathrm{C}_{49} \mathrm{H}_{82} \mathrm{O}_{6}$ & $46: 6$ & C10-C14-C22:6 \\
\hline DHA-4 & 812.6770 & $\mathrm{C}_{51} \mathrm{H}_{86} \mathrm{O}_{6}$ & $48: 6$ & C10-C16-C22:6 \\
\hline DHA-5 & 840.7081 & $\mathrm{C}_{53} \mathrm{H}_{90} \mathrm{O}_{6}$ & $50: 6$ & C12-C16-C22:6 \\
\hline DHA-6 & 868.7394 & $\mathrm{C}_{55} \mathrm{H}_{94} \mathrm{O}_{6}$ & $52: 6$ & C14-C16-C22:6 \\
\hline DHA-7 & 894.7551 & $\mathrm{C}_{57} \mathrm{H}_{96} \mathrm{O}_{6}$ & $54: 7$ & C14-C18:1-C22:6 \\
\hline DHA-8 & 896.7707 & $\mathrm{C}_{57} \mathrm{H}_{98} \mathrm{O}_{6}$ & $54: 6$ & C16-C16-C22:6 \\
\hline DHA-9 & 922.7864 & $\mathrm{C}_{59} \mathrm{H}_{100} \mathrm{O}_{6}$ & $56: 7$ & C16-C18:1-C22:6 \\
\hline DHA-10 & 950.8177 & $\mathrm{C}_{61} \mathrm{H}_{104} \mathrm{O}_{6}$ & $58: 7$ & C18-C18:1-C22:6 \\
\hline
\end{tabular}


Influence of Animal Diet on n-3 LC-PUFA Accumulation in Milk TAG

When used as a feed supplement, both RGM and WGM significantly reduced the content of nearly all TAG species that contain EPA, except EPA-17, with the RGM diet causing a greater reduction in some cases as compared with the WGM diet (Figure 4). By contrast, RGM and WGM diets did not seem to have any significant influence on the accumulation of any of the 10 TAG species containing DHA (Figure 4).

In the case of TAG containing DPA, only 1 low-abundance species (DPA-17) was promoted by both RGM and WGM, and 2 medium-abundance species (DPA-20 and DPA-21) were promoted by RGM. The level of the remaining 18 species was not modified by the diet treatment (Figure 4). Within the same dietary group, the abundance of most TAG species showed considerable variation across different cows. An example of such variation between individual cows is shown for DHA-2 (Figure 5).

It is interesting to note that strong correlation was found between different species within each class, as exemplified by the pairs EPA-11/EPA-12 and DHA-4/ DHA-5 (Figure 6). This implies that the synthesis of TAG molecules that contain n-3 LC-PUFA is highly correlated with maintenance of the relative proportion of each species. Detailed pairwise correlation between all species within each class is given in Supplemental Tables S2 to S4 (http://dx.doi.org/10.3168/jds.20159889).

When the whole data matrix (51 TAG species of 32 samples) was subjected to PCA analysis, a clear separation was observed between the control and the 2

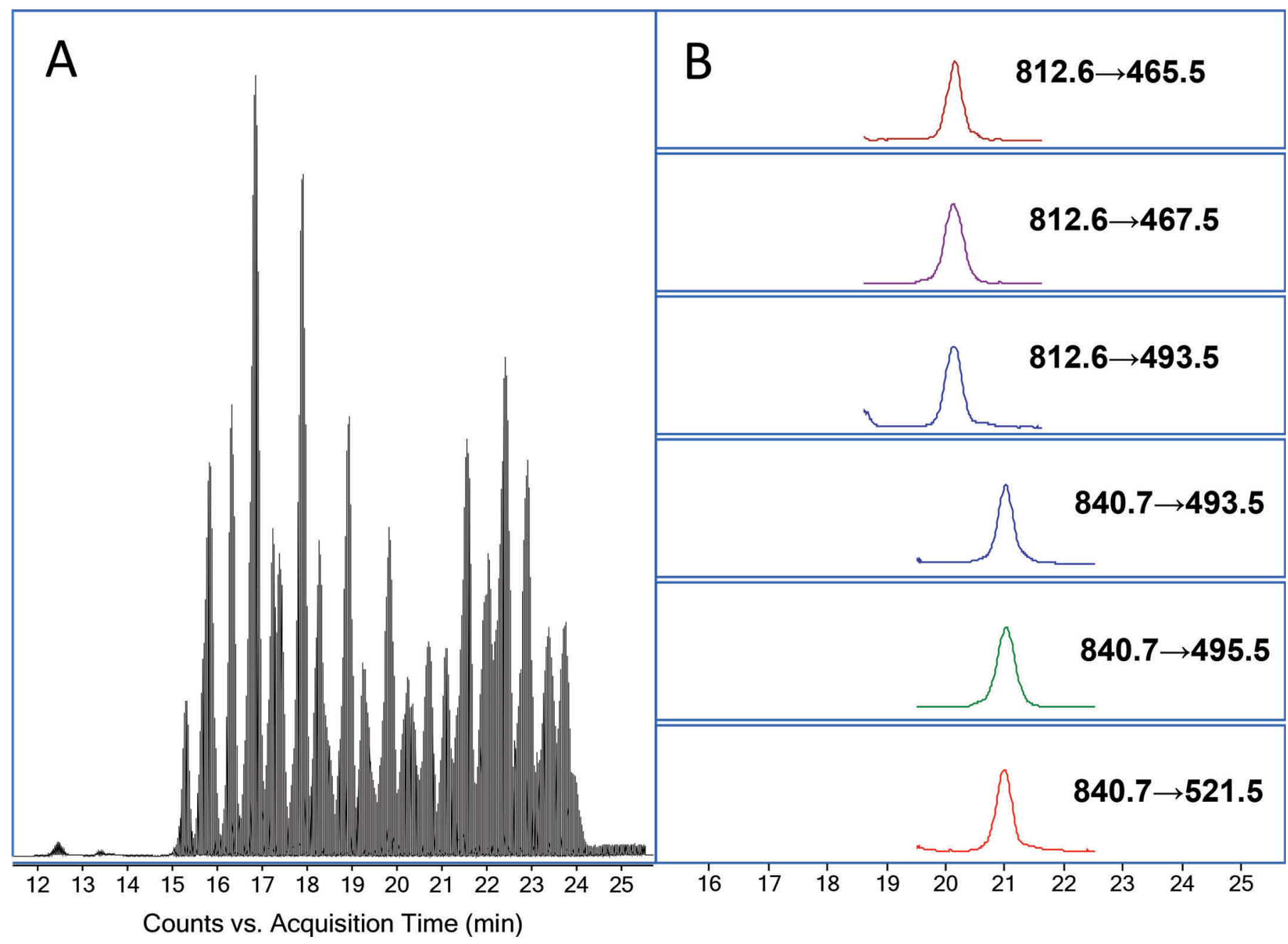

Figure 3. Total ion chromatogram of all 51 transitions (A) and 6 selected multiple reaction monitoring chromatograms of a milk sample (B). Color version available online. 

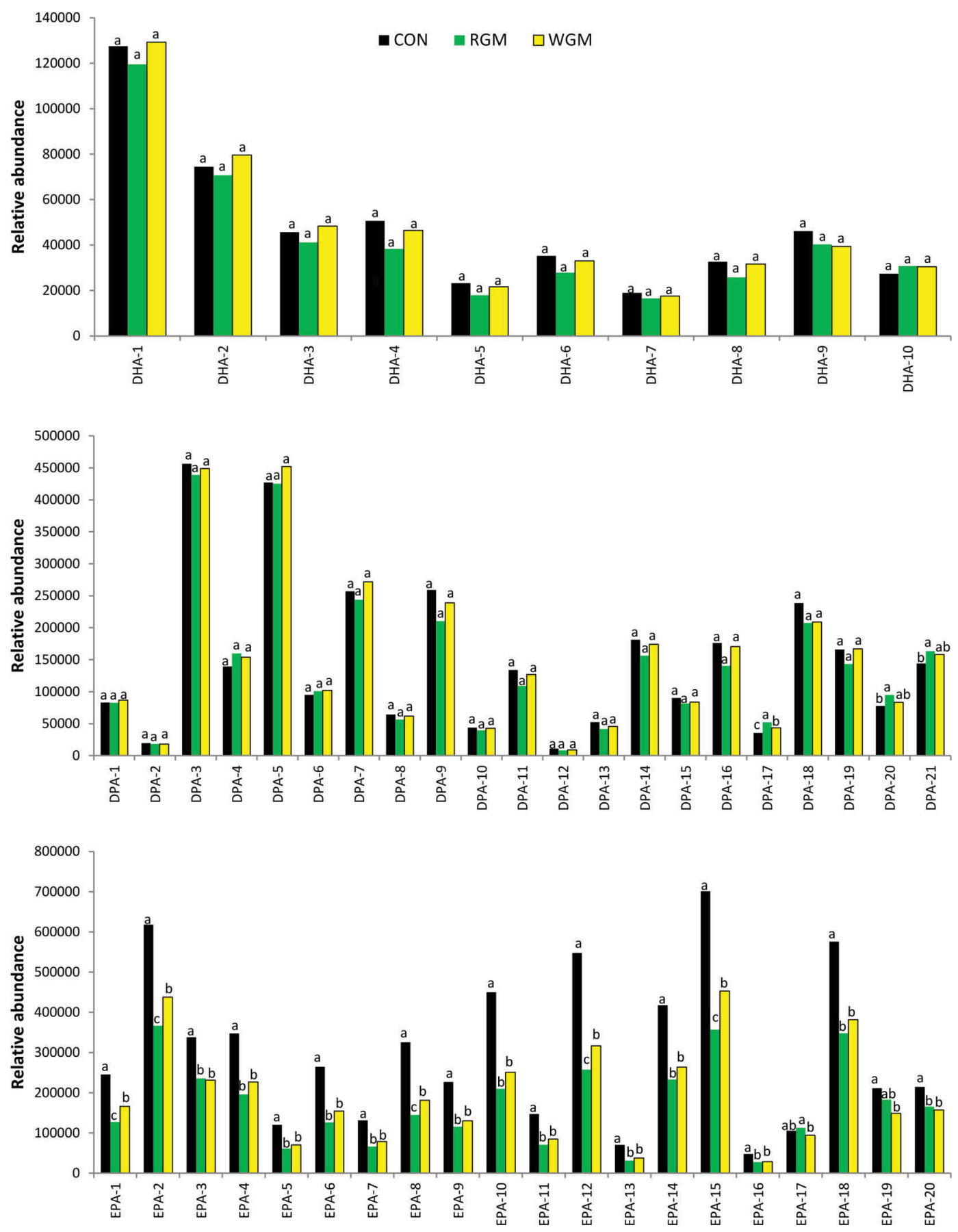

Figure 4. Effect of feed supplement on the content of n-3 long-chain PUFA-containing triacylglyceride species $(\mathrm{CON}=\mathrm{control}, \mathrm{n}=12 ; \mathrm{RGM}$ $=$ red grape marc, $\mathrm{n}=10$; WGM $=$ white grape marc, $\mathrm{n}=10)$. Within each species, columns with different letters $(\mathrm{a}-\mathrm{c})$ differ significantly $(P$ $<0.05)$. DHA = docosahexaenoic acid; DPA = docosapentaenoic acid; EPA = eicosapentaenoic acid. Color version available online.

grape marc-feeding groups, implying that the intake of grape marc has altered the distribution pattern of n-3 LC-PUFA in milk (Figure 7). However, only marginal separation was attained between the RGM and WGM diets, indicating both types of grape marc had rather similar effects in relation to n-3 LC-PUFA accumulation in milk.

The total amount of each of the 3 n-3 LC-PUFA (in the form of methyl ester) in the 3 groups of samples was measured by liquid chromatography-MS after 
transesterification of all FA-containing lipid species. The liquid chromatography-MS profile of these 3 FAME is shown in Supplementary Figure S1 (http:// dx.doi.org/10.3168/jds.2015-9889). Figure 8 shows that the total content of EPA released from all lipid species is significantly lower in samples collected from RGMand WGM-fed cows, whereas that of DHA was low and similar across the 3 groups of samples. In the case of the total DPA level, no significant difference was found between the 3 types of dietary treatment.

\section{DISCUSSION}

The n-3 LC-PUFA are dietary fats with an array of health benefits, which have been reviewed recently (Swanson et al., 2012; Abedi and Sahari, 2014). As this type of FA is typically found in fish oils, the supply may be limited by global fishery stocks. It is generally accepted that milk is a poor source of DHA and EPA, contributing insignificantly to the daily intake of around $450 \mathrm{mg}$ of EPA and DHA recommended in most countries (Kris-Etherton et al., 2009). However, a recent study suggested that milk fat may be an important source for dietary intake of EPA (van Valenberg et al., 2013). Although the overall FA profile is an important parameter for milk fat, characterization of TAG species will afford us an understanding of the biological mechanism of TAG manufacture and perhaps an un- derstanding of the physicochemical properties (such as fluidity and melting point) of milk TAG that enable them to be excreted from the alveoli. Until now, no study has detailed the molecular species composition of TAG that contain n-3 LC-PUFA and how the accumulation of these individual species is regulated in milk fat. As TAG are the dominant component of milk fat, our study focused only on the survey of TAG in the first instance.

Using the NL scan function of the triple quadrupole MS, we were able to identify over 70 precursors that afforded a NL corresponding to the mass of 1 of the $3 n-3$ LC-PUFA. These putative lipid species were subjected to 2 rounds of verification by LC-Orbitrap based on their accurate mass and MS/MS fragmentation pattern. A total of over 60 species have been confirmed to have an accurate mass expected for a TAG molecule and also have fragments corresponding to expected diacylglycerol ions and LC-PUFA acylium ions of a TAG species. Among them, 51 species were present at a level that allowed reliable quantification, and, in addition, at least a dozen other species were not included in the list because they were present at very low levels that did not allow reliable quantification.

In a study aimed at evaluating the effect of a n3-enriched diet on DHA and EPA accumulation in milk, Gastaldi et al. (2011) could not detect any TAG containing EPA in any sample and only detected 1

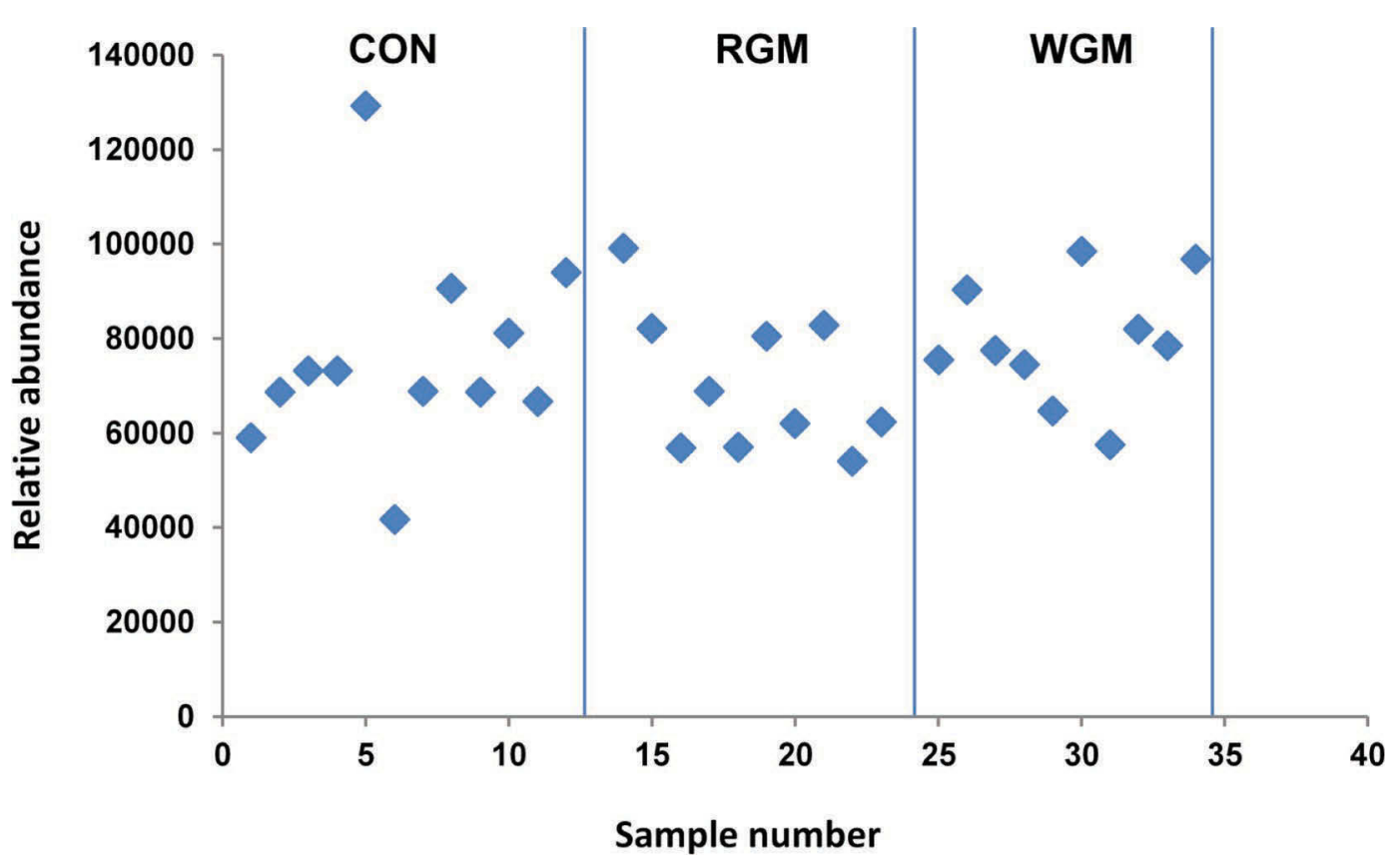

Figure 5. Variation in docosahexaenoic acid-2 content across the 32 samples. No. $1-12=$ control $(\mathrm{CON})$; no. $13-22=$ red grape marc treated (RGM); no. 23-32 = white group marc treated (WGM). Color version available online. 

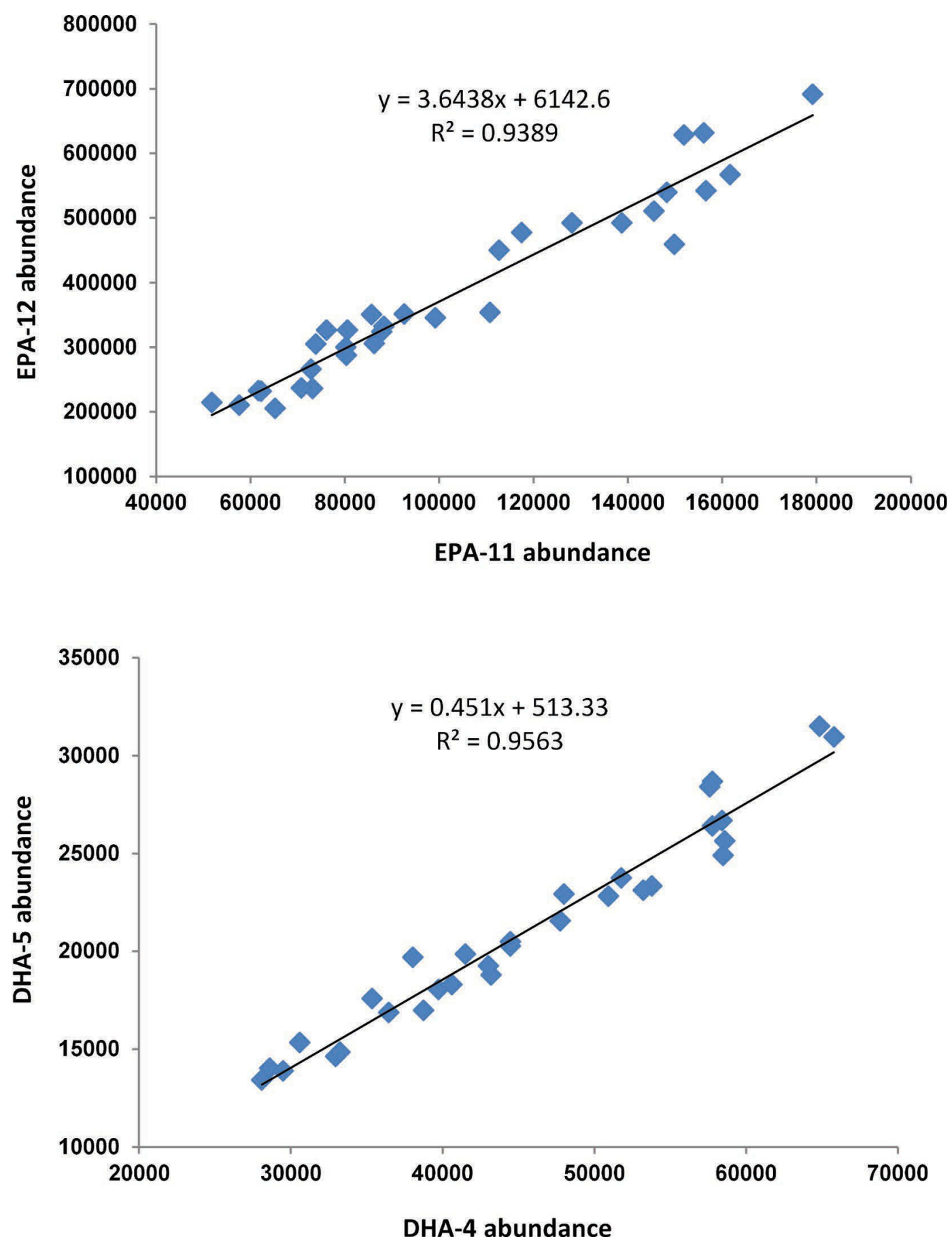

Figure 6. Significant correlation between the contents of eicosapentaenoic acid (EPA)-11/EPA-12 and docosahexaenoic acid (DHA)-4/DHA5 ( $\mathrm{n}=32$ ). Color version available online.

TAG containing DHA in enriched cow milk using the HPLC-atmospheric pressure-chemical ionization technique. Moreover, DHA was found not to be quantifiable in cow milk in several studies (O'Donnell et al., 2010; Benbrook et al., 2013). It has been our experience that TAG containing DHA appear to be prone to lipolysis, particularly in aqueous conditions and under exposure to sunlight (Moate et al., 2013). Thus it is possible that suboptimal storage of samples before analysis may account for some instances where researchers have been unable to detect TAG containing DHA in milk samples. In contrast, we were able to identify 10 TAG species 


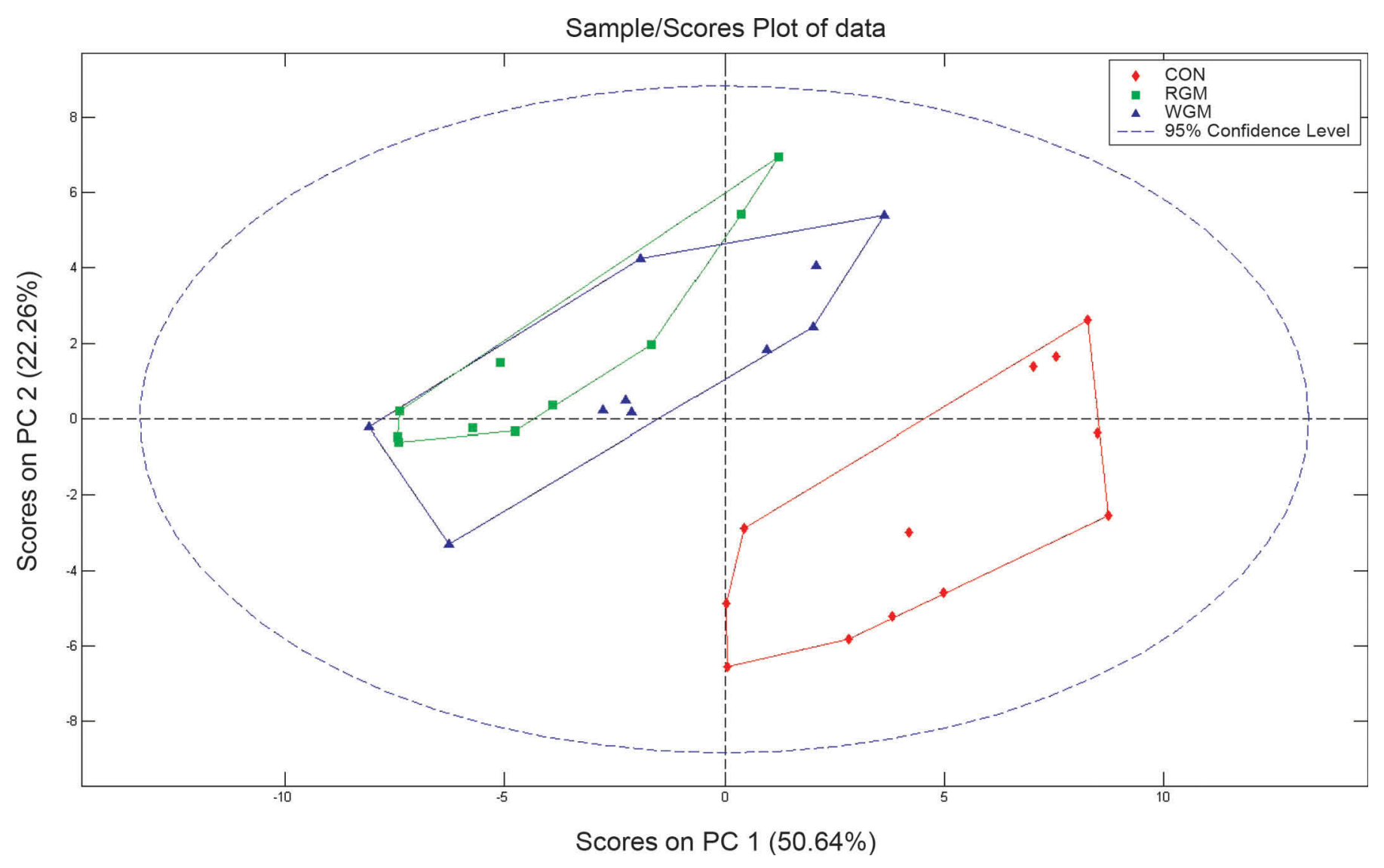

Figure 7. Principal component (PC) analysis plot of $\mathrm{PC} 1$ and $\mathrm{PC} 2$ showing unsupervised classification of 32 milk samples based on the content of $51 \mathrm{n}-3$ long-chain PUFA-containing triacylglyceride species. CON = control; RGM = red grape marc; WGM $=$ white grape marc. Color version available online.

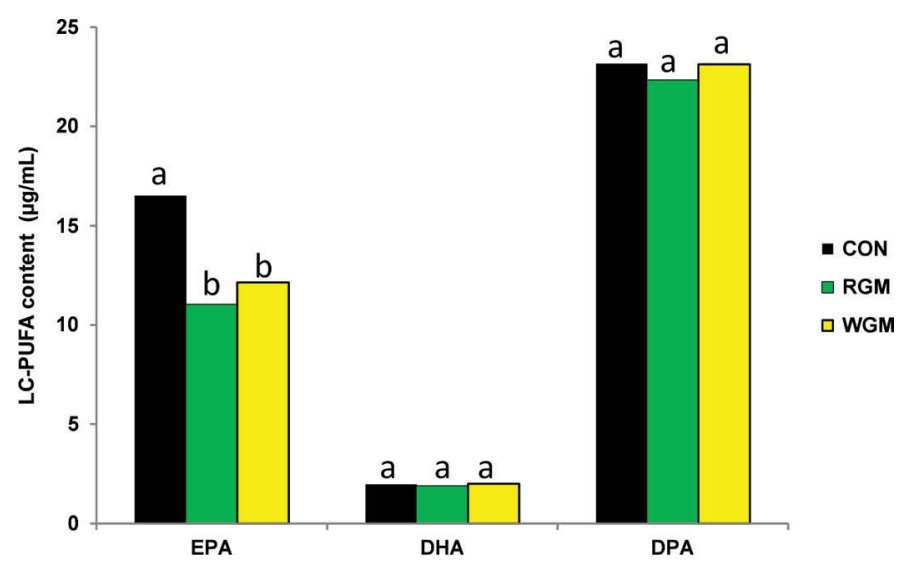

Figure 8. Effect of animal diets on milk n-3 long-chain PUFA (LCPUFA) content $(\mathrm{CON}=$ control; $\mathrm{RGM}=$ red grape marc; $\mathrm{WGM}=$ white grape marc). Within each species, columns with different letters $(\mathrm{a}, \mathrm{b})$ differ significantly $(P<0.05)$. DHA $=$ docosahexaenoic acid; DPA $=$ docosapentaenoic acid; EPA = eicosapentaenoic acid. Color version available online. containing DHA and 20 containing EPA, all present at a quantifiable level. We speculate that this discrepancy may be attributable to storage of our samples in the dark and at $-80^{\circ} \mathrm{C}$ before analysis, more concentrated samples used, or more sensitive detection techniques employed in our study. In addition, 21 TAG species containing DPA were identified in this work. This is the first systematic characterization of low-abundance TAG molecular species that contain n-3 LC-PUFA residues in bovine milk to our knowledge. In our experiment, only 1 n-3 LC-PUFA per TAG was identified. This may have occurred by chance because little n-3 LC-PUFA was present in the diet. It would be interesting to survey the n-3 TAG species present when cows are fed a diet fortified with n-3 FA.

The structure of the 51 species given in Table 1 can only be considered as the FA composition of the dominant species, because in several cases a coeluting species with a different FA composition was also found for the same parent ion, albeit at a lower abundance. Besides, the position of the FA could not be defined in this work. Fully structural elucidation of TAG remains 
a difficult task despite continuous efforts and innovative approaches (Holcapek et al., 2003; Kalo et al., 2004; Taguchi et al., 2005; Nagy et al., 2013). It is worth mentioning that isomers (with equal accurate mass, equal numbers of total carbon, and double bonds) containing different LC-PUFA are common in milk TAG, as evidenced by the fact that the 51 species are derived from 24 different chemical formulae. No attempt was made to chromatographically separate the isomers, as each interested species can be measured using a specific product ion. Due to the lack of standards, absolute quantification of TAG is not possible. As a result, a single TAG (such as tristearin) was used sometimes as external standard for quantification (Zhou et al., $2014 \mathrm{~b})$. We adopted the relative quantification strategy in this work, which allowed us to compare reliably the abundance of any particular TAG species across different samples and also compare the abundance of different species within the same n-3 LC-PUFA class in a given sample. For each of the 3 classes of TAG surveyed in this work, a large variation in abundance was observed between different species, suggesting a nonrandom distribution of these LC-PUFA in TAG. This finding is not surprising because the same observation has been reported for those more abundant FA species (Gresti et al., 1993; Fontecha et al., 2005).

When this method was applied to the characterization of milk samples following a diet treatment of animals, a significant negative effect on TAG containing EPA was observed for nearly all species after intake of either type of grape marc, whereas the same diet regimen only had minor or nil effect on species containing DHA or DPA. The total amount of EPA, DHA, and DPA methyl ester determined after transesterification confirmed the results obtained by direct quantification of individual TAG species. Our study also confirmed that when the cow diet was not fortified with DHA, milk was not an important source for DHA intake, but the significant amount of EPA and DPA (about 40 $\mathrm{mg} / \mathrm{L}$ ) could contribute substantially to human intake of n-3 FA, given that, in the absence of fatty fish consumption, the intake of n-3 FA is less than $100 \mathrm{mg} / \mathrm{d}$ (Calder, 2006).

The observation that grape marc was associated with reduction of EPA levels in milk may have 2 explanations. The simplest explanation would be that grape marc contains less EPA as compared with the fresh pasture grass that was partially replaced. However, the possibility that the conversion of $\alpha$-linolenic acid to EPA is inhibited after intake of grape marc cannot be excluded, as the proportion of EPA from direct uptake and from biological conversion are unknown. In the current study we also observed substantial variation in the content of TAG species between cows of the same dietary group. This suggests that genetic selection for animals producing higher levels of these important lipids may be possible.

\section{CONCLUSIONS}

We conducted a comprehensive survey of TAG containing n-3 LC-PUFA in milk fat using LC-LTQOrbitrap MS and LC-triple quadrupole MS, and a total of 51 TAG species that contain 1 of the 3 n-3 LC-PUFA (EPA, DHA, and DPA) were identified for the first time and their structures tentatively assigned. An LC-triple quadrupole MS method was developed, enabling quantification of the 51 TAG species in a run of $36 \mathrm{~min}$. The application of the method allowed us to reveal that both RGM and WGM, when used as a feed supplement, have adverse effects on the accumulation of EPA in milk.

\section{REFERENCES}

Abedi, E., and M. A. Sahari. 2014. Long-chain polyunsaturated fatty acid sources and evaluation of their nutritional and functional properties. Food Sci. Nutr. 2:443-463.

Abeywardena, M. Y., and G. S. Patten. 2011. Role of w3 longchain polyunsaturated fatty acids in reducing cardio-metabolic risk factors. Endocr. Metab. Immune Disord. Drug Targets 11:232-246.

Benbrook, C. M., G. Butler, M. A. Latif, C. Leifert, and D. R. Davis. 2013. Organic production enhances milk nutritional quality by shifting fatty acid composition: A United States-wide, 18-month study. PLoS ONE 8:e82429 http://dx.doi.org/10.1371/journal. pone.0082429.

Bligh, E. G., and W. J. Dyer. 1959. A rapid method of total lipid extraction and purification. Can. J. Biochem. Physiol. 37:911-917.

Calder, P. C. 2006. n-3 Polyunsaturated fatty acids, inflammation, and inflammatory diseases. Am. J. Clin. Nutr. 83:1505S-1519S.

Cheng, B., G. Wu, P. Vrinten, K. Falk, J. Bauer, and X. Qiu. 2010. Towards the production of high levels of eicosapentaenoic acid in transgenic plants: the effects of different host species, genes and promoters. Transgenic Res. 19:221-229.

Fontecha, J., H. Goudjil, J. J. Rios, M. J. Fraga, and M. Juarez. 2005. Identify of the major triacylglycerols in ovine milk fat. Int. Dairy J. 15:1217-1224.

Frelich, J., M. Slachta, O. Hanus, J. Spicka, and E. Samkova. 2009. Fatty acid composition of cow milk fat produced on low-input mountain farms. Czech J. Anim. Sci. 54:532-539.

Gastaldi, D., C. Medana, V. Giancotti, R. Aigotti, F. Dal Bello, and C. Baiocchi. 2011. HPLC-APCI analysis of triacylglycerols in milk fat from different sources. Eur. J. Lipid Sci. Technol. 113:197-207.

Gresti, J., M. Bugaut, C. Maniongui, and J. Bezard. 1993. Composition of molecular species of triacylglycerols in bovine milk fat. J. Dairy Sci. 76:1850-1869.

Haddad, I., M. Mozzon, R. Strabbioli, and N. G. Frega. 2011. Electrospray ionization tandem mass spectrometry analysis of triacylglycerols molecular species in camel milk (Camelus dromedaries). Int. Dairy J. 21:119-127.

Holcapek, M., P. Jandera, P. Zderadicka, and L. Hruba. 2003. Characterization of triacylglycerol and diacylglycerol composition of plant oils using high-performance liquid chromatography-atmospheric pressure chemical ionization mass spectrometry. J. Chromatogr. A 1010:195-215.

Kalo, P., A. Kemppinen, V. Ollilainen, and A. Kuksis. 2004. Regiospecific determination of short-chain triacylgltcerols in butter by normal-phase HPLC with on-line electrospray-tandem mass spectrometry. Lipids 39:915-928. 
Kris-Etherton, P. M., J. A. Grieger, and T. D. Etherton. 2009. Dietary reference intake for DHA and EPA. Prostaglandins Leukot. Essent. Fatty Acids 81:99-104.

Liu, Z., P. Moate, B. Cocks, and S. Rochfort. 2015. Comprehensive polar lipid identification and quantification in milk by liquid chromatography-mass spectrometry. J. Chromatogr. B Analyt. Technol. Biomed. Life Sci. 978-979:95-102.

Månsson, H. L. 2008. Fatty acids in bovine milk fat. Food Nutr. Res. http://dx.doi.org/10.3402/fnr.v52i0.1821.

Moate, P. J., W. Chalupa, R. C. Boston, and I. J. Lean. 2007. Milk fatty acids. I. Variation in the concentration of individual fatty acids in bovine milk. J. Dairy Sci. 90:4730-4739.

Moate, P. J., S. R. O. Williams, M. C. Hannah, R. J. Eckard, M. J. Auldist, B. E. Ribaux, J. L. Jacobs, and W. J. Wales. 2013. Effects of feeding algal meal high in docosahexaenoc acid on feed intake, milk production, and methane emissions in dairy cows. J. Dairy Sci. 96:3177-3188.

Moate, P. J., S. R. O. Williams, V. A. Torok, M. C. Hannah, B. E. Ribaux, M. H. Tavendale, R. J. Eckard, J. L. Jacobs, M. J. Auldist, and W. J. Wales. 2014. Grape marc reduces methane emissions when fed to dairy cows. J. Dairy Sci. 97:5073-5087.

Moghadasian, M. H. 2008. Advances in dietary enrichment with n-3 fatty acids. Crit. Rev. Food Sci. Nutr. 48:402-410.

Mori, T. A. 2006. Omega-3 fatty acids and hypertension in humans. Clin. Exp. Pharmacol. Physiol. 33:842-846.

Mottram, H. R., and R. P. Evershed. 2001. Elucidation of the composition of bovine milk fat triacylglycerols using high-performance liquid chromatography-atmospheric pressure chemical ionization mass spectrometry. J. Chromatogr. A 926:239-253.

Nagy, K., L. Sandoz, F. Destaillats, and O. Schafer. 2013. Mapping the regioisomeric distribution of fatty acids in triacylglycerols by hybrid mass spectrometry. J. Lipid Res. 54:290-305.

Nelson, K. A., and S. Martini. 2009. Increasing omega fatty acid content in cow's milk through diet manipulation: Effect on milk flavour. J. Dairy Sci. 92:1378-1386.

Nichols, P. D., J. Petrie, and S. Singh. 2010. Long-chain omega-3 oilsan update on sustainable sources. Nutrients 2:572-585.

O'Donnell, A. M., K. P. Spatny, J. L. Vicini, and D. E. Bauman. 2010 Survey of the fatty acid composition of retail milk differing in label claims based on production management practices. J. Dairy Sci. 93:1918-1925.
Penasa, M., F. Tiezzi, P. Gottardo, M. Cassandro, and M. De Marchi. 2015. Genetics of milk fatty acid groups predicted during routine data recording in Holstein dairy cattle. Livest. Sci. 173:9-13.

Petrie, J. R., P. Shrestha, X. R. Zhou, P. M. Monsour, Q. Liu, S. Belide, P. D. Nichols, and S. P. Singh. 2012. Metabolic engineering plant seeds with fish oil-like levels of DHA. PLoS ONE 7:e49165 http://dx.doi.org/10.1371/journal.pone.0049165.

Ruxton, C., S. C. Reed, M. J. A. Simpson, and K. J. Millington. 2007. The health benefits of omega-3 polyunsaturated fatty acids: a review of the evidence. J. Hum. Nutr. Diet. 20:275-285.

Sijtsma, L., and M. E. de Swaaf. 2004. Biotechnological production and application of the $\omega-3$ polyunsaturated fatty acid docosahexaenoic acid. Appl. Microbiol. Biotechnol. 64:146-153.

Slover, H. T., and E. Lanza. 1979. Quantitative analysis of food fatty acids by capillary gas chromatography. J. Am. Oil Chem. Soc. 56:933-943.

Swanson, D., R. Block, and S. A. Mousa. 2012. Omega-3 fatty acids EPA and DHA: Health benefits throughout life. Adv. Nutr. 3:1-7.

Taguchi, R., T. Houjou, H. Nakanishi, T. Yamazaki, M. Ishida, M. Imagawa, and T. Shimizu. 2005. Focus lipidomics by tandem mass spectrometry. J. Chromatogr. B Analyt. Technol. Biomed. Life Sci. 823:26-36.

Taylor, A. G., and C. Savage. 2006. Fatty acid composition of New Zealand green-lipped mussels, Perna canaliculus: Implications for harvesting for n-3 extracts. Aquaculture 261:430-439.

van Valenberg, H. J. F., K. A. Hettinga, J. Dijkstra, H. Bovenhuis, and E. J. M. Feskens. 2013. Concentration of n-3 and n- 6 fatty acids in Dutch bovine milk fat and their contribution to human dietary intake. J. Dairy Sci. 96:4173-4181.

Wu, G., M. Truksa, N. Datla, P. Vrinten, J. Bauer, T. Zank, P. Cirpus, E. Heinz, and X. Qiu. 2005. Stepwise engineering to produce high yields of very long-chain polyunsaturated fatty acids in plants. Nat. Biotechnol. 23:1013-1017.

Zhou, Q., B. Gao, X. Zhang, Y. Xu, H. Shi, and L. Yu. 2014a. Chemical profiling of triacylglycerols and diacylglycerols in cow milk fat by ultra-performance convergence chromatography combined with a quadrupole time-of-flight mass spectrometry. Food Chem. 143:199-204

Zhou, X. R., D. L. Callahan, P. Shrestha, Q. Liu, J. R. Petrie, and S. P. Singh. 2014b. Lipidomics analysis of Arabidopsis seed genetically engineered to contain DHA. Front. Plant Sci. 5:419. 